\title{
La importancia del resumen en un artículo científico
}

\section{The importance of abstract in a scientific article}

El resumen de un artículo científico nos permite comunicar en forma rápida y significativa el contenido de un documento. Para la norma ISO un resumen constituye la representación abreviada y precisa de ese contenido, sin críticas ni interpretaciones. Por otra parte, ANSI/NISO conceptualiza al resumen como una presentación objetiva y breve de un documento o de una exposición oral.

El resumen puede determinar si un documento se ajusta a nuestros intereses o si nos resulta relevante para los objetivos que guían nuestra búsqueda. Sólo después de su lectura buscaremos acceder al texto completo y seguramente lo citaremos en nuestro trabajo. Del mismo modo, los resúmenes propios pueden otorgar mayor visibilidad a nuestras producciones ya que potencian la posibilidad de que otros autores nos citen. Como expresa Repiso "la cita se ha asociado desde muy temprano con la calidad de los trabajos, pues es un elemento hipertextual que proviene de un reconocimiento de autoridad que hace el autor de un trabajo a otros trabajos previos."

Además de las características que posee un buen resumen como la objetividad, la precisión y la claridad, este tipo de texto posee funciones específicas: por un lado, es utilizado como elementos de recuperación de la información en las bases de datos bibliográficas; por otro, constituye un elemento de difusión del documento, y finalmente, es el que conduce a la selección de un trabajo y a su correspondiente citación.

Los resúmenes anticipan el texto principal, y dado que muchas veces es lo único que los lectores leen también lo sustituyen. Son un reflejo en miniatura del artículo completo, ya que en ellos se sintetiza el propósito del trabajo, los métodos, los resultados más importantes y las principales conclusiones. Los resúmenes constituyen la materia prima de los sistemas los índices de citas, por ejemplo, para el conocido Index Medicus, actualmente accesible a través de base de datos MEDLINE/PubMed.

La International Committee of Medical Journal Editors (ICMJE), en sus recomendaciones para la realización, elaboración de informes, edición y publicación de trabajos académicos en revistas médicas, lo cual podría ser aplicado a cualquier ciencia biológica, brinda los lineamientos básicos para

Revista Methodo: Investigación Aplicada a las Ciencias Biológicas. Facultad de Medicina. Universidad Católica de Córdoba. Jacinto Ríos 571 Bo Gral. Paz. X5004FXS. Córdoba. Argentina. Tel.: (54) 3514517299 / Correo: methodo@ucc.edu.ar / Web: methodo.ucc.edu.ar | EDITORIAL Rev. Methodo 2021;6(2):59-60. 
la incorporación de resúmenes en artículos de investigación, revisiones sistemáticas y metaanálisis. La Revista Methodo, en sintonía con dichas recomendaciones, dentro de las instrucciones para autores, solicita que los resúmenes contengan:

- Introducción breve del estado del conocimiento sobre el tema a investigar y el objetivo de la investigación.

- Población y métodos para señalar dónde y cuándo se realizó el estudio, el diseño del trabajo y la población estudiada. Definir la variable principal de resultado.

- Resultados de la variable principal y descripción del resto de los resultados obtenidos y su significación estadística.

- Conclusiones puntuales, con relación a los objetivos planteados y los resultados obtenidos.

Para finalizar, es importante destacar que, para el editor de una revista, el resumen puede ayudar a decidir la aceptación o rechazo de un trabajo; para el autor, un buen resumen puede conducir a que otros seleccionen el trabajo y lo citen, mientras que, para el revisor, el resumen debe constituir un elemento más de evaluación para garantizar la calidad del artículo.

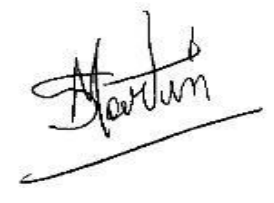

Mgter. Sandra Gisela Martín Directora. Sistema de Bibliotecas Universidad Católica de Córdoba

Bibliografía.

- ANSI/NISO Z39.14-1997 (R2015) Guidelines for abstracts https://www.niso.org/publications/ansiniso-z3914-1997-r2015guidelines-abstracts

- ICMJE. (2019). Recommendations for the Conduct, Reporting, Editing, and Publication of Scholarly Work in Medical Journals http://www.icmje.org/icmje-recommendations.pdf

-ISO 214-1976 Abstracts for publications and documentation https://www.iso.org/standard/4084.html

-Repiso, R. (2020, abril 27). La cita como indicador de calidad. Revista Comunicar. Escuela de Autores.

https://doi.org/10.3916/escuela-de-autores-129.

\section{(c) (1) \&(0) BY NC SA}

Revista Methodo: Investigación Aplicada a las Ciencias Biológicas. Facultad de Medicina. Universidad Católica de Córdoba. Jacinto Ríos 571 Bo Gral. Paz. X5004FXS. Córdoba. Argentina. Tel.: (54) 3514517299 / Correo: methodo@ucc.edu.ar / Web: methodo.ucc.edu.ar | EDITORIAL Rev. Methodo 2021;6(2):59-60. 